\title{
THE NUMBER OF COMPACT SUBSETS OF A TOPOLOGICAL SPACE
}

\author{
D. K. BURKE AND R. E. HODEL
}

\begin{abstract}
Results are obtained which give an upper bound on the number of compact subsets of a topological space in terms of other cardinal invariants. The countable version of the main theorem states that an $\kappa_{1}$ compact space with a point-countable separating open cover has at most $2^{k_{0}}$ compact subsets.
\end{abstract}

1. Introduction. Let $w, L, d, c, \chi$, and $\psi$ denote the following standard cardinal functions: weight, Lindelöf degree, density, cellularity, character, and pseudo-character. (For definitions, see Juhász [11].) If $\phi$ is a cardinal function, then $h \phi$ is the hereditary version of $\phi$; i.e., $h \phi(X)=\sup \{\phi(Y): Y \subseteq X\}$. The collection of all compact subsets of a topological space $X$ is denoted by $\mathscr{K}(X)$.

Among the best-known theorems in cardinal functions are those which give an upper bound on the cardinality of a space in terms of other cardinal invariants. In this paper we will be concerned with the following such results.

I. (Pospišil [17]) If $X$ is Hausdorff, then $|X| \leqslant 2^{2^{d(X)}}$

II. (de Groot [6]) If $X$ is Hausdorff, then $|X| \leqslant 2^{h L(X)}$.

III. (Arhangel' skii [2]) If $X$ is Hausdorff, then $|X| \leqslant 2^{L(X) \cdot \chi(X)}$.

IV. (Hajnal-Juhász [9]) If $X$ is Hausdorff, then $|X| \leqslant 2^{c(X) \cdot \chi(X)}$.

V. (Hajnal-Juhász [9]) If $X$ is $T_{1}$, then $|X| \leqslant 2^{h c(X) \cdot \psi(X)}$.

A key step in the proof of recent theorems obtained by Arhangel'skii [1], Hodel [10], and Juhász [12] is to determine the number of compact subsets of a topological space. This suggests the general problem of finding an upper bound on the cardinality of $\mathcal{K}(X)$ in terms of other cardinal invariants. A particularly natural question to ask is whether or not " $X$ " can be replaced by " $K(X)$ " in the inequalities in I-V. In this paper we show that this replacement is possible in I and II but not in III and IV. We do not know if the replacement is possible in $\mathrm{V}$, although it is consistent in the countable case for Hausdorff spaces. In $\$ 4$ we prove what is perhaps the main theorem in this paper. The countable version of this result states that the number of compact subsets of an $\boldsymbol{\aleph}_{1}$-compact space with a point-countable separating open cover is $\leqslant 2^{N_{0}}$.

Throughout this paper $\mathfrak{m}$ and $\mathfrak{n}$ denote cardinal numbers with $\mathfrak{m}$ infinite; $\mathfrak{m}^{+}$is the smallest cardinal greater than $\mathfrak{m} ; \alpha$ and $\beta$ denote ordinal numbers.

Received by the editors May 13, 1975.

AMS (MOS) subject classifications (1970). Primary 54A25; Secondary 54D30.

Key words and phrases. Number of compact sets, density, Lindelöf degree, cellularity, $\kappa_{1}$ compact, point-countable separating open cover. 
Unless otherwise stated, no separation axioms are assumed; however, regular spaces are always $T_{1}$.

We need several cardinal functions in addition to $w, L, d, c, \chi$, and $\psi$. For a $T_{1}$ space $X$ let $\psi K(X)=\aleph_{0} \cdot \mathfrak{n}$, where $\mathfrak{n}$ is the smallest cardinal such that every compact subset of $X$ is the intersection of $\leqslant \mathfrak{n}$ open sets. Let $\psi C(X)$ be the cardinal function obtained by replacing "compact" by "closed" in the definition of $\psi K(X)$. Note that $\psi K(X) \leqslant \psi C(X)$ and $\psi K(X) \leqslant h L(X)$ whenever $X$ is Hausdorff.

The discreteness character of a topological space $X$, denoted $\Delta(X)$, is $\aleph_{0} \cdot \mathfrak{n}$, where $\mathfrak{n}=\sup \{|\mathscr{F}|: \mathscr{F}$ is a discrete collection of nonempty closed sets in $X\}$. For the class of $T_{1}$ spaces this cardinal function extends the concept of $\boldsymbol{\kappa}_{1}$ compactness ( $=$ every uncountable set has a limit point) to higher cardinality; see $[10]$.

Let $X$ be a set, let $\delta$ be a cover of $X$. The cover $\delta$ is said to be separating if given any two distinct points $p$ and $q$ in $X$, there is some $S$ in $\delta$ such that $p \in S, q \notin S$. For $p$ in $X$, the order of $p$ with respect to $\delta$, denoted ord $(p, \delta)$, is the cardinality of the set $\{S$ in $\delta: p \in S\}$. Now assume $X$ is a $T_{1}$ space. The point separating weight of $X$, denoted psw $(X)$, is $\boldsymbol{\kappa}_{0} \cdot \mathfrak{n}$, where $\mathfrak{n}$ is the smallest cardinal such that $X$ has a separating open cover $\mathcal{S}$ with ord $(p, \mathcal{S}) \leqslant \pi$ for all $p$ in $X$. Note that $X$ has a point-countable separating open cover if and only if psw $(X)=\aleph_{0}$.

2. I and II. In this section we show that " $X$ " can be replaced by " $\mathcal{K}(X)$ " in the inequalities in I and II.

THEOREM 2.1. If $X$ is a Hausdorff space, then $|\mathcal{K}(X)| \leqslant 2^{2^{d(X)}}$.

Proof. Let $d(X)=\mathfrak{m}$; let $D$ be a dense subset of $X$ with $|D| \leqslant \mathfrak{m}$. The open collection $\mathcal{S}=\left\{(\bar{E})^{0}: E \subseteq D\right\}$ has cardinality $\leqslant 2^{\mathrm{m}}$; and, using the Hausdorff hypothesis and the denseness of $D$, one can check that $\delta$ is a separating cover of $X$. Let $\delta_{1}$ be all finite unions of elements of $\delta$, and let $\delta_{2}$ be all intersections of elements of $\delta_{1}$. Clearly $\left|\mathcal{S}_{2}\right| \leqslant 2^{2^{\mathrm{m}}}$, and so the proof is complete if we can show that $\mathscr{K}(X) \subseteq \delta_{2}$. Let $K$ be a compact subset of $X$. For each $p$ in $X$ with $p \notin K$, there is some $S_{p}$ in $\delta_{1}$ such that $K \subseteq S_{p}$ and $p$ $\notin S_{p}$. Clearly $K=\bigcap\left\{S_{p}: p \in(X-K)\right\}$, and so $K \in \delta_{2}$.

Corollary 2.2. Let $X$ be a separable Hausdorff space. Then the number of compact subsets of $X$ is $\leqslant 2^{2^{\kappa_{0}}}$.

REMARK 2.3. See [11, p. 64] for an example of a Hausdorff space of density $m$ in which the number of closed sets is $2^{2^{2 m}}$. If $X$ is a regular space, then $w(X) \leqslant 2^{d(X)}$ and so the number of closed subsets of $X$ is $\leqslant 2^{2^{d(X)}}$.

The following notation will be used in the next theorem and in $\S 4$. Let $E$ be a set, let $\mathcal{Q}$ be a collection of sets. Then $\mathscr{P}_{\mathfrak{m}}(E)=\{A \subseteq E:|A| \leqslant \mathfrak{m}\}$ and $\mathcal{Q}_{\mathfrak{m}}=\{G: G$ the union of $\leqslant \mathfrak{m}$ elements of $\mathscr{Q}\}$.

LEMMA 2.4 (ŠAPIROVSKII [18]). Let $थ$ be an open cover of a topological space $X$, let $\mathfrak{m}=h c(X)$. Then there is some $G$ in $\mathcal{Q}_{\mathfrak{m}}$ and some $A$ in $\mathscr{P}_{\mathfrak{m}}(X)$ such that $X=G \cup \bar{A}$.

THEOREM 2.5. If $X$ is a Hausdorff space, then $|\mathcal{K}(X)| \leqslant 2^{h c(X) \cdot \psi K(X)}$. 
Proof. Let $\mathrm{m}=h c(X) \cdot \psi K(X)$; then by $\mathrm{V},|X| \leqslant 2^{\mathrm{m}}$. For each $p$ in $X$ let $\vartheta_{p}$ be a collection of open neighborhoods of $p$, closed under finite intersections, such that $\left|\mathscr{U}_{p}\right| \leqslant 2^{\mathfrak{m}}$ and $\cap\left\{\bar{U}: U\right.$ in $\left.\mathscr{U}_{p}\right\}=\{p\}$. Let $\mathcal{Q}=\cup\left\{\mathscr{U}_{p}\right.$ : $p$ in $X\}$, let $\mathcal{C}=\left\{G \cup \bar{A}: G\right.$ in $\mathcal{Q}_{\mathfrak{m}}, A$ in $\left.\mathscr{P}_{\mathfrak{m}}(X)\right\}$, and let $\mathcal{E}=\left\{E: E\right.$ in $\left.\mathcal{C}_{\mathfrak{m}}\right\}$. Now $|\mathcal{E}| \leqslant 2^{\mathfrak{m}}$, and so the proof is complete if we can show that the complement of each compact subset of $X$ is in $\mathscr{E}$.

Let $K \subseteq X$ be compact. Since $\psi K(X) \leqslant \mathfrak{m},(X-K)=\bigcup\left\{F_{\alpha}: 0 \leqslant \alpha\right.$ $<\mathfrak{m}$, where each $F_{\alpha}$ is a closed subset of $X$. Fix $\alpha<\mathfrak{m}$. For each $p$ in $F_{\alpha}$, use the compactness of $K$ to obtain some $U_{p}$ in $\mathscr{U}_{p}$ such that $U_{p} \cap K=\phi$. Apply Sapirovskii's Lemma to $F_{\alpha}$ and $\left\{U_{p}: p\right.$ in $\left.F_{\alpha}\right\}$ to obtain $G_{\alpha}$ in $\mathcal{U}_{\mathfrak{m}}$ and $A_{\alpha}$ in $\mathscr{P}_{\mathfrak{m}}\left(F_{\alpha}\right)$ such that $F_{\alpha} \subseteq G_{\alpha} \cup \overline{A_{\alpha}}$. Let $C_{\alpha}=G_{\alpha} \cup \overline{A_{\alpha}}$, and note that $C_{\alpha}$ is in $e$ and $C_{\alpha} \cap K=\phi$. Clearly $(X-K)=\cup\left\{C_{\alpha}: 0 \leqslant \alpha<\mathfrak{m}\right\}$, and so $(X-K)$ is in $\mathcal{E}$.

Corollary 2.6. Let $X$ be a Hausdorff space which hereditarily satisfies the $C C C$ and in which every compact set is a $G_{\delta}$. Then the number of compact subsets of $X$ is $\leqslant 2^{\aleph_{0}}$.

Corollary 2.7. If $X$ is a Hausdorff space, then $|\mathcal{K}(X)| \leqslant 2^{h L(X)}$. In particular, if $X$ is a Hausdorff, hereditarily Lindelöf space, then the number of compact subsets of $X$ is $\leqslant 2^{\kappa_{0}}$.

Corollary 2.8. If $X$ is a Hausdorff space, then $|\mathcal{K}(X)| \leqslant 2^{\Delta(X) \psi C(X)}$. In particular, if $X$ is a Hausdorff, $\aleph_{1}$-compact space in which every closed set is a $G_{\delta}$, then the number of compact subsets of $X$ is $\leqslant 2^{\aleph_{0}}$.

Proof. Let $\mathfrak{m}=\Delta(X) \cdot \psi C(X)$. Then $h c(X) \leqslant \mathfrak{m}$ by a result of Hodel [10], and so $h c(X) \cdot \psi K(X) \leqslant \mathrm{m}$. Hence $|\mathcal{K}(X)| \leqslant 2^{\mathfrak{m}}$ by 2.5 .

REMARK 2.9. Hajnal and Juhász [8] have proved the consistency, for each $\mathrm{m}$, of a regular space $X$ with $h L(X)=\mathfrak{m}$ and $w(X)=2^{2^{m}}$. Thus 2.5, 2.7, and 2.8 cannot be extended to "number of closed sets is $\leqslant 2^{\mathfrak{m}}$."

EXAMPLE 2.10. A Hausdorff, hereditarily separable, non-Lindelöf space in which every closed set is $a G_{\delta}$. The example is due to the referee, and utilizes a construction of Hajnal and Juhász [7]. Let $X$ be a set of real numbers of cardinality $\boldsymbol{N}_{1}$, and let $\mathscr{B}$ be a countable base for the subspace Euclidean topology on $X$. Let $X$ be enumerated as $\left\{x_{\alpha}: 0 \leqslant \alpha<\omega_{1}\right\}$, and for each $\alpha<\omega_{1}$ let $U_{\alpha}=\left\{x_{\beta}: \beta \leqslant \alpha\right\}$. Then $\mathscr{B} \cup\left\{U_{\alpha}: 0 \leqslant \alpha<\omega_{1}\right\}$ is a subbase for a topology $\sigma$ on $X$ which is Hausdorff, hereditarily separable, and not Lindelöf. The following lemma, due to the referee, shows that every closed subset of $(X, \mathcal{T})$ is a $G_{\delta}$.

LeMma 2.11. Let $\Re$ be a countable base for a metric topology on $X$ and let $\mathcal{Q}$ be a topology on $X$ such that every proper open set is countable. If $\sigma$ is the topology on $X$ generated by $\mathscr{B} \cup$ Q, then every open subset of $(X, \mathcal{T})$ is an $F_{\sigma}$.

Proof. Let $H \in$ T; then we can write $H=G \cup D$, where $G$ is the interior of $H$ relative to the topology with base $\mathscr{B}$, and $D=H-G$. Since $G$ is an $F_{\sigma}$ set, it suffices to show that $D$ is countable. Suppose not. For each $x \in D$, choose $B_{x} \in \mathscr{B}$ and $U_{x} \in \mathcal{Q}$ such that $x \in B_{x} \cap U_{x} \subseteq H$. Since $\mathscr{B}$ is countable, there is a fixed $B \in \mathscr{B}$ and an uncountable set $D^{\prime} \subseteq D$ such that 
$B_{x}=B$ for all $x \in D^{\prime}$. Now $\cup_{x \in D^{\prime}} U_{x}=X$, and so $B \subseteq H$. Hence $D^{\prime} \subseteq B$ $\subseteq G$, a contradiction.

3. III, IV, and V. We begin this section by giving an example of a compact, Hausdorff, first countable, separable space in which the number of compact subsets is $2^{2^{\kappa_{0}}}$. This example shows that " $X$ " cannot be replaced by " $\mathcal{K}(X)$ " in the inequalities in III and IV. (The authors would like to thank I. Juhász for calling their attention to this example; see p. 68 in [11].) Let $I^{*}$ be the top and bottom line of the lexicographically ordered square with the order topology, and let $X=I^{*} \times I^{*}$. It is well known that $X$ is compact, Hausdorff, first countable, and separable. Moreover, $X$ contains a discrete subspace of cardinality $2^{\aleph_{0}}$ (see [11]), and from this fact it follows that $X$ contains $2^{2^{\aleph_{0}}}$ open sets. Thus, $X$ contains $2^{2^{k_{0}}}$ closed and hence compact sets.

We do not know if " $X$ " can be replaced by " $\mathcal{K}(X)$ " in the inequality in $\mathrm{V}$, although such a replacement is consistent in the countable case for Hausdorff spaces. The general problem seems quite difficult.

Theorem 3.1. Assume $M A+\neg C H$. Let $X$ be a Hausdorff space which hereditarily satisfies the CCC and has countable pseudo-character. Then the number of compact subsets of $X$ is $\leqslant 2^{\aleph_{0}}$.

Proof. By $\mathrm{V},|X| \leqslant 2^{\aleph_{0}}$, and so we need only show that each compact subset of $X$ is the closure of a countable subset of $X$. But any compact subset of $X$ is a compact, Hausdorff, first countable, CCC space, and by a result of Juhász [11], MA $+\neg \mathrm{CH}$ implies that such a space is separable.

REMARK 3.2. Šapirovskii [19] has recently proved that $d(X) \leqslant h c(X)^{+}$ whenever $X$ is a compact Hausdorff space. Using this result, one easily obtains the following theorem. If $X$ is a Hausdorff space, then $|\mathcal{K}(X)| \leqslant 2^{(\psi(X) \cdot h c(X))^{+}}$. Theorem 3.1 follows from this result, since MA $+\neg \mathrm{CH}$ implies $2^{\aleph_{0}}=2^{\aleph_{1}}$ [13].

4. A new inequality. Several lemmas are needed to prove the main result of this section.

LemMa 4.1 (BURKe [3]). Let $\mathfrak{m}$ be an infinite cardinal, let $\left\{E_{t}: t\right.$ in $\left.A\right\}$ be a collection of sets with $|A|>2^{\mathfrak{m}}$ and $\left|E_{t}\right| \leqslant \mathfrak{m}$ for all $t$ in $A$. Suppose $E_{t}$ $=E_{t}^{\prime} \cup E_{t}^{\prime \prime}$ and $E_{t}^{\prime} \cap E_{t}^{\prime \prime}=\phi$ for all $t$ in $A$. Then there is some $B \subseteq A,|B|$ $>2^{\mathfrak{m}}$, such that if $s$ and $t$ are in $B$, then $E_{s}^{\prime} \cap E_{t}^{\prime \prime}=\phi$.

Lemma 4.2 (SEe [10]). Let $X$ be a topological space with $\Delta(X) \leqslant \mathfrak{m}$, let $\Re$ be an open cover of $X$ such that ord $(p, \Re) \leqslant \mathrm{m}$ for all $p$ in $X$. Then there is a subcollection of $\Re$ of cardinality $\leqslant \mathfrak{m}$ which covers $X$.

LEMMA 4.3 (MišČENKo [5], [ 15]). Let $K$ be a set, let $m$ be an infinite cardinal, and let $\delta$ be a collection of sets such that ord $(p, \delta) \leqslant \mathfrak{m}$ for all $p$ in $K$. Then the cardinality of the set of all finite minimal covers of $K$ by elements of $\delta$ does not exceed $\mathrm{m}$.

THeOREM 4.4. If $X$ is $T_{1}$, then $|\mathcal{F}(X)| \leqslant 2^{\Delta(X) \cdot \operatorname{psw}(X)}$.

Proof. Let $\mathfrak{m}=\Delta(X) \cdot$ psw $(X)$. First we prove that $|X| \leqslant 2^{\mathfrak{m}}$. Suppose $|X|>2^{\mathfrak{m}}$. Let $\delta$ be a separating open cover of $X$ such that ord $(p, \delta) \leqslant \mathfrak{m}$ for all $p$ in $X$. For $p$ in $X$ let $\varsigma_{p}=\{S$ in $\delta: p \in S\}$. Now 


$$
X-\{p\}=\cup\left\{X-S: S \text { in } \delta_{p}\right\},
$$

$\Delta(X-S) \leqslant \mathfrak{m}$ for each $S$ in $\delta_{p}$, and $\left|\delta_{p}\right| \leqslant \mathfrak{m}$, so $\Delta(X-\{p\}) \leqslant \mathfrak{m}$. Let $\Re_{p}=\{S$ in $\delta: p \notin S\}$. Then $\Re_{p}$ is an open cover of $X-\{p\}$ such that for all $q$ in $X-\{p\}$, ord $\left(q, \Re_{p}\right) \leqslant \mathfrak{m}$. By Lemma 4.2 there is a subcollection $\mathscr{W}_{p}$ of $\Re_{p}$ with $\left|\mathcal{W}_{p}\right| \leqslant m$ which covers $X-\{p\}$. Note that $\mathcal{W}_{p} \cap \delta_{p}=\phi$. By Lemma 4.1, there is some $Y \subseteq X$ with $|Y|>2^{\mathfrak{m}}$ such that if $p$ and $q$ are in $Y$, then $\mho_{p} \cap \delta_{q}=\phi$. Let $p$ and $q$ be distinct points of $Y$. Pick $S$ in $\mho_{p}$ such that $q \in S$. Now $S \in \delta_{q}$, and hence $\mho_{p} \cap \delta_{q} \neq \phi$, a contradiction. Thus we have $|X| \leqslant 2^{\mathrm{m}}$.

Next we prove $|\mathcal{K}(X)| \leqslant 2^{\mathfrak{m}}$. Since $|X| \leqslant 2^{\mathfrak{m}}$ and ord $(p, \delta) \leqslant \mathfrak{m}$ for all $p$ in $X$, it follows that $|\delta| \leqslant 2^{\mathfrak{m}}$. Let $\mho$ be all finite unions of elements of $\delta$, and let $\mathcal{G}$ be all intersections of $\leqslant \mathfrak{m}$ elements of $\mho$. Now $|\mathcal{G}| \leqslant 2^{\mathfrak{m}}$, and so the proof is complete if we can show that $\mathcal{K}(X) \subseteq \mathcal{G}$. Given a compact subset $K$ of $X$, let $\left\{\delta_{\alpha}: 0 \leqslant \alpha<\mathfrak{m}\right\}$ be all finite minimal covers of $K$ by elements of $\mathcal{S}$ (use Miščenko's Lemma), and for each $\alpha<m$ let $W_{\alpha}=\cup \delta_{\alpha}$. Then $K$ $=\cap\left\{W_{\alpha}: 0 \leqslant \alpha<\mathfrak{m}\right\}$ and so $K \in \mathcal{G}$.

Corollary 4.5. Let $X$ be an $\aleph_{1}$-compact space with a point-countable separating open cover. Then the number of compact subsets of $X$ is $\leqslant 2^{\aleph_{0}}$. In particular, $|X| \leqslant 2^{\aleph_{0}}$.

REMARK 4.6. In the proof of 4.4 , the fact that $|\mathcal{S}| \leqslant 2^{\mathfrak{m}}$ can also be established using a technique based on ideas of M. E. Rudin [4] and Sapirovskiı [18]. Indeed, let $\delta$ and $X$ be as in 4.4, and note that it suffices to show that $d(X) \leqslant 2^{\mathfrak{m}}$. Construct transfinite sequences $\left\{D_{\alpha}: 0 \leqslant \alpha<\mathfrak{m}^{+}\right\}$ and $\left\{\delta_{\alpha}: 0<\alpha<\mathfrak{m}^{+}\right\}$such that:

(1) $\left|D_{\alpha}\right| \leqslant 2^{\mathfrak{m}}$ and $\left|\delta_{\alpha}\right| \leqslant 2^{\mathfrak{m}}$;

(2) $\cup_{\beta<\alpha} D_{\beta} \subseteq D_{\alpha} \subseteq X$

(3) $\mathcal{S}_{\alpha}=\left\{S \in \delta: S \cap\left(\cup_{\beta<\alpha} D_{\beta}\right) \neq \phi\right\}$;

(4) if $G \in\left(\delta_{\alpha}\right)_{\mathfrak{m}}$ and $G \neq X$, then $D_{\alpha} \cap(X-G) \neq \phi$.

Let $D=\cup\left\{D_{\alpha}: 0 \leqslant \alpha<\mathrm{m}^{+}\right\}$; clearly $|D| \leqslant 2^{\mathrm{m}}$, and so it remains to prove that $D$ is dense. Let $p \in X$, but suppose $p \notin \bar{D}$. Let $\Re=\{S$ in $\delta: S \cap \bar{D}$ $\neq \phi, p \notin S\}$. Since $\mathcal{S}$ is a separating open cover of $X$, $\Re$ covers $\bar{D}$. Now $\Delta(\bar{D}) \leqslant \mathfrak{m}$, so by 4.2 , there is a subcollection $\Re_{1}$ of $\Re$ with $\left|\Re_{1}\right| \leqslant \mathfrak{m}$ which covers $\bar{D}$. If $S \in \Re_{1}$, then $S \cap D \neq \phi$. Since $D=\bigcup\left\{D_{\alpha}: 0 \leqslant \alpha\right.$ $\left.<\mathfrak{m}^{+}\right\}$and $\left|\Re_{1}\right| \leqslant \mathfrak{m}$, it follows that $\Re_{1} \subseteq \delta_{\alpha}$ for some $\alpha<\mathfrak{m}^{+}$. Let $G$ $=\cup \Re_{1}$. Now $G \in\left(\delta_{\alpha}\right)_{\mathfrak{m}}$ and $G \neq X$, so by (4), $(X-G) \cap D_{\alpha} \neq \phi$. This contradicts the fact that $\Re_{1}$ covers $\bar{D}$.

REMarK 4.7. The above technique can also be used to prove de Groot's result that $|X| \leqslant 2^{h L(X)}$ for $X$ Hausdorff. (This observation has also been made by R. Pol [16].) The necessary modifications are sketched as follows. Let $\mathfrak{m}=h L(X)$. For each $p$ in $X$ let $\vartheta_{p}$ be a collection of open neighborhoods of $p$ with $\left|\vartheta_{p}\right| \leqslant m$ such that $\cap \mathcal{Q}_{p}=\{p\}$. Construct transfinite sequences $\left\{D_{\alpha}: 0 \leqslant \alpha<\mathfrak{m}^{+}\right\}$and $\left\{\mathscr{Q}_{\alpha}: 0<\alpha<\mathrm{m}^{+}\right\}$such that:

(1) $\left|D_{\alpha}\right| \leqslant 2^{\mathfrak{m}}$ and $\left|\vartheta_{\alpha}\right| \leqslant 2^{\mathfrak{m}}$;

(2) $\cup_{\beta<\alpha} D_{\beta} \subseteq D_{\alpha} \subseteq X$

(3) $\mathcal{U}_{\alpha}=\cup\left\{\vartheta_{p}: p \in \cup_{\beta<\alpha} D_{\beta}\right\}$;

(4) if $G \in\left(\mathcal{U}_{\alpha}\right)_{\mathfrak{m}}$ and $G \neq X$, then $D_{\alpha} \cap(X-G) \neq \phi$. 
Then $X=\cup\left\{D_{\alpha}: 0 \leqslant \alpha<\mathfrak{m}^{+}\right\}$and so $|X| \leqslant 2^{\mathfrak{m}}$.

EXAMPLE 4.8. A regular, Lindelöf space $X$ with a $\sigma$-disjoint base which has $2^{2^{k_{0}}}$ closed sets. The example, due to Michael [14], is obtained as follows. Let $Y$ be a subset of the unit interval of cardinality $2^{\aleph_{0}}$, all of whose compact subsets are countable. Let $X$ be the unit interval retopologized so that $Y$ is discrete. (Specifically, the topology is the collection of all sets of the form $U \cup V$, where $U$ is an ordinary open set of the interval and $V$ is a subset of $Y$.) This example shows that 4.5 cannot be extended to "number of closed sets is $\leqslant 2^{\aleph_{0}}$

\section{REFERENCES}

1. A. V. Arhangel'skiri, On hereditary properties, General Topology and Appl. 3 (1973), 38-46.

2. On the cardinality of bicompacta satisfying the first axiom of countability, Dokl. Akad. Nauk SSSR 187 (1969), 967-970 = Soviet Math. Dokl. 10 (1969), 951-955. MR 40 \#4922.

3. D. K. Burke, A note on R. H. Bing's example G, Topology Conf. Virginia Polytechnic Institute and State University, Lecture Notes in Math., vol. 375, Springer-Verlag, New York, 1974, pp. 47-52.

4. H. H. Corson and E. Michael, Metrizability of certain countable unions, Illinois J. Math. 8 (1964), 351-360. MR 30 \#562.

5. V. V. Filippov, On feathered paracompacta, Dokl. Akad. Nauk SSSR 178 (1968), 555-558 = Soviet Math. Dokl. 9 (1968), 161-164. MR 37 \#3519.

6. J. de Groot, Discrete subspaces of Hausdorff spaces, Bull. Acad. Polon. Sci. Sér. Sci. Math. Astronom. Phys. 13 (1965), 537-544. MR 35 \# 956.

7. A. Hajnal and I. Juhász, On hereditarily $\alpha$-Lindelöf and hereditarily $\alpha$-separable spaces, Ann. Univ. Sci. Budapest Eötvös Sect. Math. 11 (1968), 115-124. MR 39 \#2124.

8. - A consistency result concerning hereditarily $\alpha$-Lindelöf spaces, Acta Math. Acad. Sci. Hungar. (to appear).

9. __ Discrete subspaces of topological spaces, Nederl. Akad. Wetensch. Proc. Ser. A 70 = Indag. Math. 29 (1967), 343-356. MR 37 \#769.

10. R. E. Hodel, On a theorem of Arhangel'skii concerning Lindelöf p-spaces, Canad. J. Math. 27 (1975), 459-468.

11. I. Juhász, Cardinal functions in topology, Math. Centre Tracts, no. 34, Mathematisch Centrum, Amsterdam, 1971. MR 49 \#4778.

12. - A generalization of nets and bases (preprint).

13. D. A. Martin and R. M. Solovay, Internal Cohen extensions, Ann. Math. Logic 2 (1970), no. 2, 143-178. MR 42 \#5787.

14. E. Michael, The product of a normal space and a metric space need not be normal, Bull. Amer. Math. Soc. 69 (1963), 375-376. MR 27 \#2956.

15. A. S. Miščenko, Spaces with point-countable bases, Dokl. Akad. Nauk SSSR 144 (1962), 985-988 = Soviet Math. Dokl. 3 (1962), 855-858. MR 25 \# 1537.

16. R. Pol, Short proofs of two theorems on cardinality of topological spaces, Bull. Acad. Polon. Sci. Sér. Sci. Math. Astronom. Phys. 22 (1974), 1245-1249.

17. B. Pospišil, Mohutnost prostoru s hustou cásti dané mohutnosti, Časopis Pěst. Mat. Fys. 67 (1937/38), 89-96.

18. B. Sapirovskiĭ, On discrete subspaces of topological spaces: weight, tightness, and Suslin number, Dokl. Akad. Nauk SSSR 202 (1972), 779-782 = Soviet Math. Dokl. 13 (1972), 215-219. MR 45 \# 1100.

19. - Canonical sets and character. Density and weight in compact spaces, Dokl. Akad. Nauk SSSR 218 (1974), 58-61 = Soviet Math. Dokl. 15 (1974), 1282-1287.

Department of Mathematics, Miami University, Oxford, Ohio 45056 